Research paper

\title{
Skin compatibility of cyclodextrins and their derivatives: a comparative assessment using a corneoxenometry bioassay
}

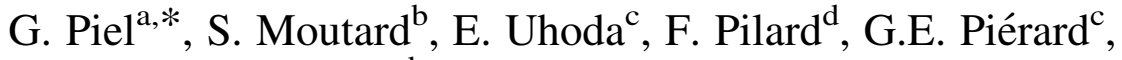 \\ B. Perly ${ }^{\mathrm{b}}$, L. Delattre ${ }^{\mathrm{a}}$, B. Evrard ${ }^{\mathrm{a}}$ \\ ${ }^{a}$ Laboratory of Pharmaceutical Technology, Department of Pharmacy, University of Liège, Liège, Belgium \\ ${ }^{\mathrm{b}}$ Laboratory of Molecular Chemistry, Gif-sur-Yvette, France \\ ${ }^{\mathrm{c}}$ Department of Dermatopathology, University Hospital, Liège, Belgium \\ ${ }^{\mathrm{d}}$ Laboratory of Glucides, University of Amiens, Amiens, France
}

Received 22 September 2003; accepted in revised form 15 December 2003

\begin{abstract}
Few studies have been performed to assess the risk of skin damage by cyclodextrins (CD) and they have yielded contradictory results. The present study was conducted using the corneoxenometry bioassay on human stratum corneum to compare the skin compatibility of $\mathrm{CD}$ currently used in pharmaceutical preparations ( $\beta C D, \gamma C D$, Rameb, Dimeb, Trimeb, HP- $\beta C D$ and HP- $\gamma C D$ ) and that of new amphiphilic CD derivatives, namely, the phospholipidyl-CD (DMPE-Dimeb and DMPE-Trimeb). All the tested CD were well tolerated by the stratum corneum at a concentration of 5\%. However, inter-individual reactivity was larger for DMPE-Dimeb, suggesting a more aggressive trend for this compound. Cutaneous Index of Mildness values obtained confirm that Dimeb is able to extract some skin components and shows that DMPE-Dimeb performs similarly.

(C) 2004 Elsevier B.V. All rights reserved.
\end{abstract}

Keywords: Corneoxenometry; Skin compatibility; Cyclodextrins; Phospholipidyl-cyclodextrins; Stratum corneum

\section{Introduction}

For more than 20 years, cyclodextrins (CD) have attracted interest owing to their possible applications in a wide range of fields from fundamental research to industry. Their practical use mainly arose from the capability of their inclusion complexes to modify the chemical reactivity and physical characteristics of the guest molecules. Many chemically modified CD have been synthesized in order to improve or modify some of their properties. Amphiphilic $\mathrm{CD}$ are especially capable of forming by themselves or in association with other amphiphiles or guest molecules, stable supramolecular assemblies well recognized as efficient drug carriers, such as micelles, vesicles or nanospheres. It has been also shown that they can be inserted

\footnotetext{
* Corresponding author. Laboratory of Pharmaceutical Technology, Department of Pharmacy, University of Liège, CHU, Tour 4, Bat. B36, 1 av. de l'Hôpital, B-4000 Liège, Belgium. Tel.: +32-43664307; fax: $+32-43664302$.

E-mail address: geraldine.piel@ulg.ac.be (G. Piel).
}

through their hydrophobic part into lipidic systems such as liposomes, leaving the $\mathrm{CD}$ cavities outside and available to include active guests. These supramolecular assemblies represent new promising vectors since they combine the size specificity of CD and the properties of organized bilayers as molecular transporters [1].

With this scope in mind, amphiphilic CDs carrying a phospholipidyl chain (the dimyristoylphosphatidylethanolamine, DMPE), the DMPE-dimethyl- $\beta C D$ (DMPE-Dimeb) and the DMPE-trimethyl- $\beta C D$ (DMPE-Trimeb), have been synthesized to mimic, as closely as possible, natural membranes. The novel glyco-conjugates have been fully characterized for dermatological delivery of xenobiotics with low aqueous solubility (Fig. 1).

Many articles describe the use of CD in dermatological preparations, but few studies describe their compatibility with the skin and in particular with the stratum corneum (SC). It is well known that CD may interact with some components of the skin, but contradictory results have been reported so far [2]. For instance, Dimeb is known to extract cholesterol and triglycerides from powdered hide and from 


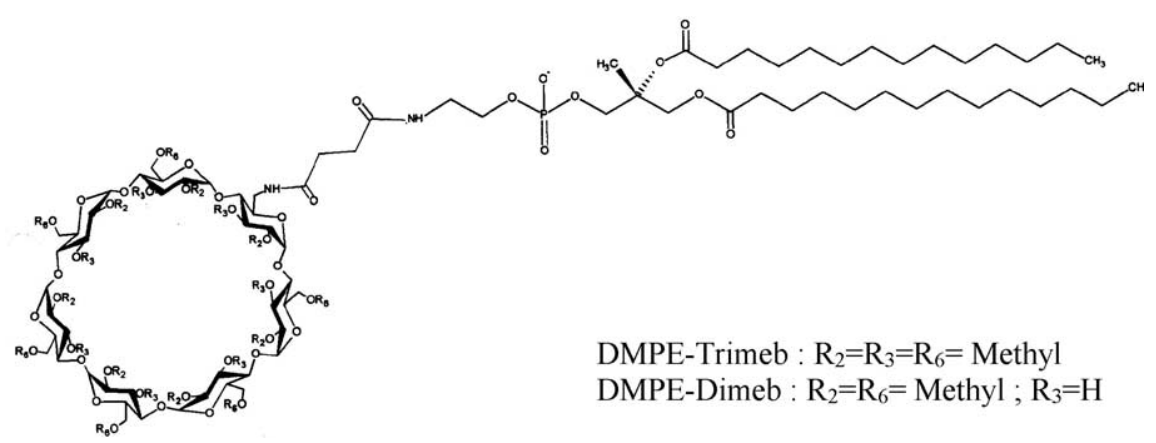

Fig. 1. Chemical structure of the phospholipidyl-cyclodextrin: DMPE-Dimeb and DMPE-Trimeb.

rabbit skin in vitro, a process that may alter the barrier function of the SC and eventually may lead to increased drug absorption [3]. It is generally assumed that the SC barrier is disrupted through intercellular lipid removal and protein alteration. In such a case, particular attention should be directed toward the possible irritation effects of $\mathrm{CD}$ on the skin [4]. The lack of toxicological data hampers pharmaceutical applications of CD [2].

The aim of this study was to compare the skin irritation potential of the new DMPE-CD derivatives with that of native $\mathrm{CD}(\beta \mathrm{CD}$ and $\gamma \mathrm{CD})$ and $\mathrm{CD}$ derivatives currently used for pharmaceutical applications including the randomly methylated $\beta C D$ (Rameb), the dimethylated $\beta C D$ (Dimeb), the trimethylated $\beta C D$ (Trimeb) and the hydroxypropylated $\beta C D$ and $\gamma \mathrm{CD}$ (HP- $\beta C D$ and $\mathrm{HP}-\gamma \mathrm{CD})$. For this purpose, the corneoxenometry bioassay was performed on human SC.

Corneoxenometry, named after the terms corneocyte, xenobiotic and metry, was designed for testing chemicals biologically harmful to the SC [4-7]. It is a variant of corneosurfametry initially developed to rate the aggressiveness of surfactants on human SC [5, 8]. The substrate used for testing is normal human SC collected by skin surface stripping (SSS) using cyanoacrylate adhesive and terephtalate polymer sheets. The harvested material corresponding to a uniform sheet of SC is submitted to the action of xenobiotics ex vivo (immersion into a solution). Once the contact time is over, samples are dried and stained with basic fuchsin-toluidine blue dye solution (polychrome multiple stain, PMS). Normal SC avidly binds anionic dyes because corneocytes are covered by acidophilic, positively charged loci. Once altered by a series of chemicals or other threats, corneocytes exhibit distinct staining properties by cationic dyes. One of the most convenient mixtures associates basic fuchsin and toluidine blue which stain selectively the altered stratum corneum. The interaction can be explained by three complementary mechanisms:

- The treatment extracts lipids of corneocytes, increasing the access of PMS to cell membrane proteins.

- The treatment denatures proteins of corneocytes increasing the number of negative charges fixing PMS.
- The treatment is adsorbed on corneocytes forming a bridge fixing PMS.

Whatever the hypothesis, quantifying the changes in the colour of the stained CSSS was considered to be an indicator of the amount of sites exposed on the surface of corneocytes by xenobiotics eliciting removal of intercellular lipids and denaturation of proteins [5].

Accurate colour expression depends on three primary elements: hue (rainbow colours), corresponding to the dominant wavelength, lightness (brightness) and saturation (vividness). The continuum in colour hues is classically pictured as a colour wheel clearly exhibiting the complementary hues (red-green, yellow-blue, ...). In terms of physics lightness represents the colour value, and saturation is called chroma. A three-dimensional representation of colours is obtained by the combination of the hue colour wheel with a vertical axis of value and a horizontal axis of chroma from the centre. Quantitative and repetitive measurements are only reliable when objective measurements are made. This can be achieved by reflectance tristimulus Commission Internationale de l'Eclairage (CIE) colorimetry. The CIELAB colour space system is now widely used [9].

Value $L^{*}$ is expressed on a scale ranging from 0 for black to 100 for white. Hue and chroma are expressed by combining chromacities $a^{*}$ and $b^{*}$. In the colour wheel (Fig. 2), $a^{*}$ and $b^{*}$ indicate two orthogonal colour axes, with $a^{*}$ being the red $(+100)$ and green $(-100)$ axis and $b^{*}$ the yellow $(+100)$-blue $(-100)$ axis. The positive section of axis $a^{*}$ (red) and the positive section of axis $b^{*}$ (yellow) are used to measure skin colours. Chroma $C^{*}$ is described by the equation, $C^{*}=\left(a^{* 2}+b^{* 2}\right)^{1 / 2}$ [9]. Corneoxenometry data are expressed by the colorimetric index of mildness (CIM) which corresponds to the difference between $L^{*}$ and chroma $C^{*}$ values of each CSSS test sample. Previous studies have shown decreased CIM values with increasing irritancy of test products. Irritant products have CIM values lower than 30; those that are very irritant have a CIM value lower than 10 and even negative value. The CIM water value (mildness reference) is around 64 while that of sodium laurylsulfate, a reference irritating product, may be as low as $5-15[8]$. 


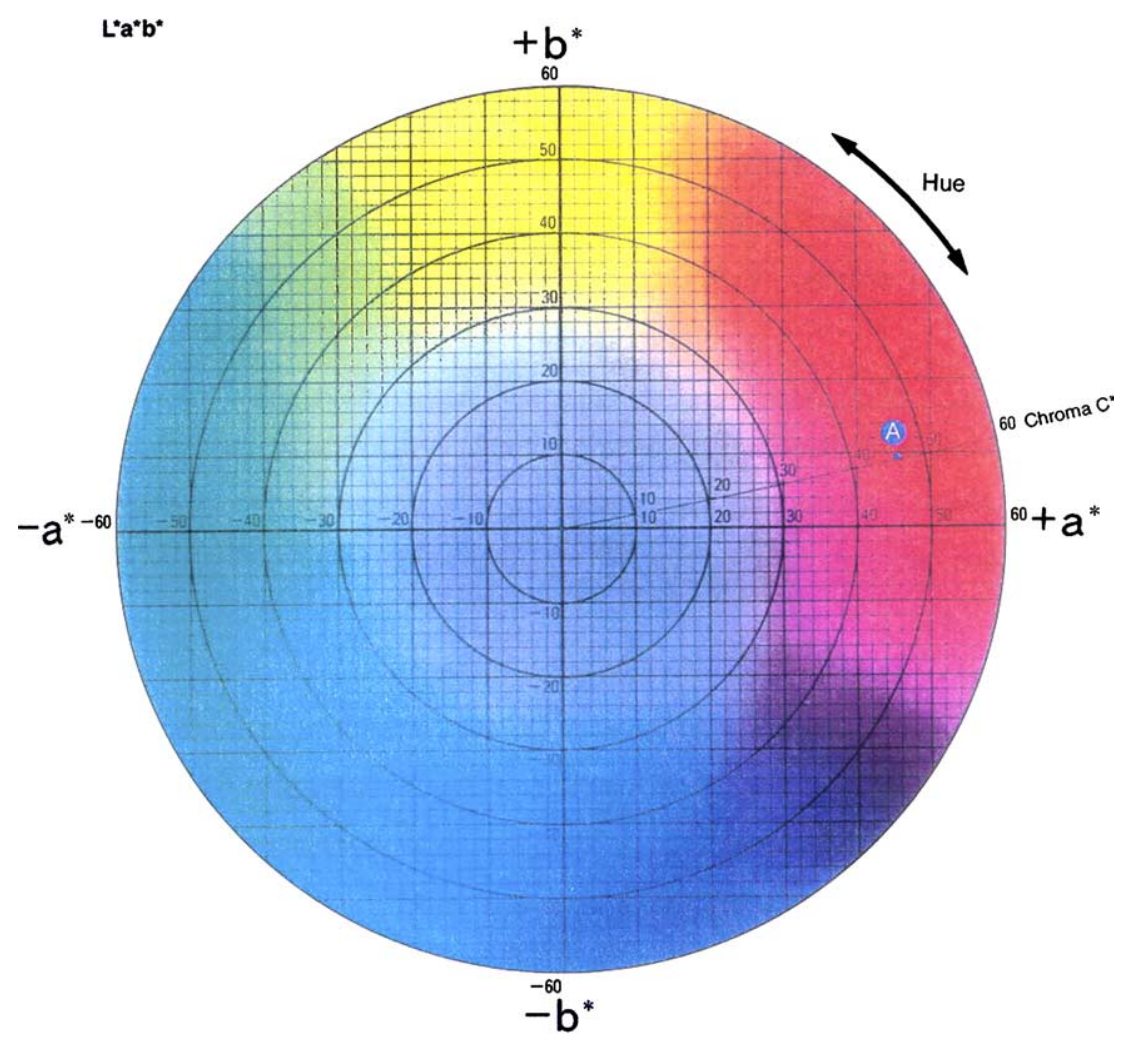

Fig. 2. Colour wheel in the plane of the $a^{*}$ and $b^{*}$ axis.

Results obtained by corneoxenometry or corneosurfametry show good correlations with those of other in vitro tests and in vivo studies [6].

\section{Material and methods}

\subsection{Materials}

$\beta C D$ was obtained from CNI (Neuilly-sur-Seine, France), $\gamma \mathrm{CD}, \mathrm{HP}-\gamma \mathrm{CD}$ and Rameb were obtained from Wacker Chemie GmbH (Munchen, Germany), Dimeb and Trimeb were from Cyclolab (Budapest, Hungary) and HP$\beta C D$ from Janssen Biotech (Olen, Belgium).

DMPE-Dimeb and DMPE-Trimeb were synthesized following the method described by Moutard [10].

\subsection{Experimental design}

CSSS were harvested from the ventral forearms of five healthy volunteers using a cyanoacrylate adhesive (Superglue 3, Loctite) and terephthalate polymer sheets (Melinex $\mathrm{O}, \mathrm{ICI}$ plastic division).

Samples were immersed into $5 \%$ (W/V) CD aqueous solutions for $2 \mathrm{~h}$. at room temperature $\left(20^{\circ} \mathrm{C}\right)$ in a closed environment to prevent evaporation of the test products. Due to its low aqueous solubility, $\beta C D$ was tested at $1.5 \%$ (W/V). Once the contact time was over, samples were thoroughly rinsed under running tap water. After air-drying, they were stained for $3 \mathrm{~min}$ by PMS. After removal of the excess dye and abundant rinsing with water, the samples were dried.

The resulting corneocyte alterations were quantified using reflectance colorimetry. Samples were placed on a white tile (Minolta reference plate) and a colour reflectance meter (Chroma Meter CR 200, Minolta, Osaka, Japan) was used to record luminancy $L^{*}$ and Chroma $C^{*}$.

For each series of evaluations, medians and ranges of CIM values were calculated. Statistical evaluations were performed using the paired non-parametric Friedman test followed by the Dunn's paired test. A $P$-value lower than 0.05 was considered as reaching statistical significance.

\section{Results and discussion}

Corneoxenometry is a predictive bioassay testing the compatibility of xenobiotics with the SC. Any disruption in the lipid bilayers of the SC and any protein denaturation of the corneocytes, a process that may reduce the barrier function of the SC and may induce irritation, is revealed by controlled colorimetric means. The effect of organic solvents, penetration enhancers and barrier creams were tested by corneoxenometry with relevant predictive value $[4,6,7]$. In this study, the corneoxenometry suggested that all tested CD are globally well tolerated by the skin because CIM medians values were above the threshold value of 30 for skin mildness (Fig. 3). Overall, no significant differences 


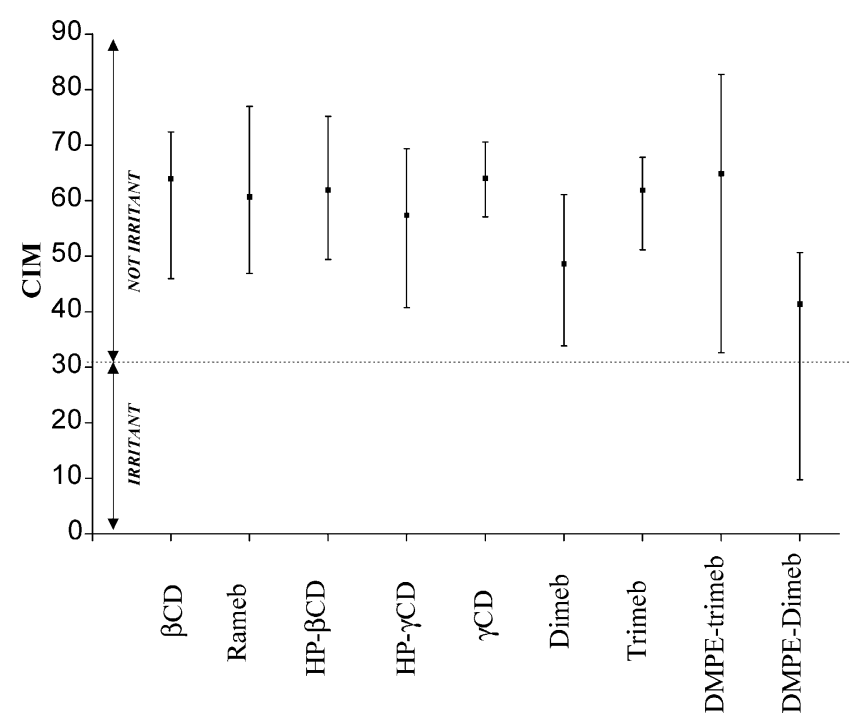

Fig. 3. Corneosurfametry bioassay. Medians and ranges of the colorimetric index of mildness (CIM) of 9 cyclodextrins tested on human stratum corneum $(n=5)$. CIM is inversely correlated with irritancy of cyclodextrins.

were yielded between most CD. The two exceptions were DMPE-Dimeb and to a lesser extent Dimeb, which exhibited significantly lower CIM values than those of most other CD. No significant difference was observed between Dimeb and DMPE-Dimeb. However, the large inter-individual CIM variability for DMPE-Dimeb predicts that some individuals suffer from reduced compatibility with this compound. Subjects complaining of sensitive skin might be those reacting with the lowest CIM values [8].

The present corneoxenometry data are in line with results suggesting that Dimeb extracts components of the skin (cholesterol, triglyceride) [3]. They are also consistent with the results obtained by DSC showing that Dimeb affected the endothermic peak transition of isolated human SC whereas no noticeable changes were observed for the SC treated with HP- $\beta C D$. Other studies have shown that HP$\beta C D$ had limited specificity for cholesterol and triglycerides [3]. The present study suggests that the phospholipidylDimeb derivative also extracts components of the skin to the same extent as Dimeb since their CIM values are similar. This means that DMPE-Dimeb could retain full capacity to include some xenobiotics, which is of considerable importance for potential applications.

This study confirms that most of the tested CD are well tolerated with a good safety profile following topical application. However, a minority of individuals might feel uncomfortable with DMPE-Dimeb.

In conclusion, all tested $\mathrm{CD}(\beta \mathrm{CD}, \gamma \mathrm{CD}$, Rameb, Trimeb, DMPE-Trimeb, HP- $\beta C D$, HP- $\gamma C D$, DMPEDimeb and DMPE-Trimeb) appear to be well tolerated by the skin. However Dimeb and DMPE-Dimeb are less mild than the others. Dimeb and the new phospholipidyl-CD derivative, DMPE-Dimeb could be used as a skin absorption enhancer because the alteration of the SC may lead to increased drug absorption.

\section{Acknowledgements}

G. Piel is a postdoctoral researcher supported by the FNRS, Brussels, Belgium. E. Uhoda is a research assistant supported by the Region Wallonne, Namur, Belgium

\section{References}

[1] S. Lesieur, D. Charon, P. Lesieur, C. Ringard, V. Muguet, D Duchene, D. Wouessidjewe, Phase behavior of fully hydrated DMPCamphiphilic cyclodextrin systems, Chem Phys Lipids 106 (2000) $127-144$.

[2] T. Loftsson, M. Masson, Cyclodextrins in topical drug formulations: theory and practice, Int J Pharm 225 (2001) 15-30.

[3] T. Irie, K. Uekama, Pharmaceutical application of cyclodextrins. III. Toxicological issues and safety evaluation, J Pharm Sci 86 (1997) $147-162$.

[4] V. Goffin, C. Letawe, G.E. Piérard, Effect of organic solvents on normal human stratum corneum. Evaluation by corneoxenometry bioassay, Dermatology 195 (1997) 321-324.

[5] G.E. Piérard, V. Goffin, C. Piérard-Franchimont, Corneosurfametry: a predictive assessment of the interaction of personal-care cleansing products with the human stratum corneum, Dermatology 189 (1994) $152-156$

[6] V. Goffin, C. Piérard-Franchimont, G.E. Piérard, Shielded corneosurfametry and corneoxenometry: novel bioassays for the assessment of skin barrier products, Dermatology 196 (1998) 434-437.

[7] V. Goffin, F. Henry, C. Piérard-Franchimont, G.E. Piérard, Penetration enhancers assessed by corneoxenometry, Skin Pharmacol Appl Skin Physiol 13 (2000) 280-284.

[8] V. Goffin, C. Piérard-Franchimont, G.E. Piérard, Sensitive skin and stratum corneum reactivity to household cleaning products, Contact Dermat 34 (1996) 81-85.

[9] G.E. Piérard, EEMCO guidance for the assessment of skin colour, J Eur Acad Dermatol Venerol 10 (1998) 1-11.

[10] S. Moutard, B. Perly, P. Gode, G. Demailly, F. Djedaïni-Pilard, Novel glycolipids based on cyclodextrins, J Inclu Phenom Macrocyclic Chem 44 (2002) 317-322. 